\title{
Validation of the short version of the Van Lieshout Test in an Italian population with cervical spinal cord injuries: a cross-sectional study
}

\author{
Anna Berardi ${ }^{1}$ - Alessio Biondillo ${ }^{1}$ Maria Auxiliadora Màrquez $^{2} \cdot$ Rita De Santis $^{3} \cdot$ Giovanni Fabbrini $^{4}$ • \\ Marco Tofani $\mathbb{D}^{1} \cdot$ Donatella Valente ${ }^{5} \cdot$ Giovanni Galeoto $\mathbb{B}^{6}$
}

Received: 20 July 2018 / Revised: 13 November 2018 / Accepted: 14 November 2018 / Published online: 14 December 2018

(c) International Spinal Cord Society 2018

\begin{abstract}
Study design Psychometric study.

Objective To validate the Italian version of the Van Lieshout Test Short Version (VLT-SV) with a spinal cord injury population.

Setting Three Italian spinal units.

Methods The Italian version of the VLT-SV (VLT-SV-IT) was administered to a sample of people with cervical spinal cord injuries (C-SCI) and the test-retest was performed. Reliability was assessed using Cronbach's alpha for internal consistency and the intraclass correlation coefficient for repeatability assessment (test-retest). Pearson's correlation coefficient was calculated for concurrent validity with the Italian version of the Jebsen-Taylor Hand Function Test (JTHFT) and for construct validity with the Italian version of the Spinal Cord Injury Independence Measure (SCIM III).

Results The VLT-SV-IT was administered to 61 individuals and all psychometric properties were significant: Cronbach's alpha was 0.95 (left hand and right hand) and the intraclass correlation coefficient for test-retest reliability was 0.90 for the right hand, the left hand, and the total score. Pearson's correlation coefficient of the VLT-SV-IT with the JTHFT was significant, while the correlation with SCIM III was not. The obtained values are considered acceptable and consistent with international guidelines.

Conclusions The VLT-SV-IT was shown to be a reliable and valid assessment tool for measuring hand function in the Italian population with C-SCI. This result suggests that it could be used as a starting point for hand therapy and to assist in clinical decision-making regarding treatment policy.
\end{abstract}

Giovanni Galeoto

giovanni.galeoto@uniroma1.it

"Sapienza" University of Rome, Rome, Italy

2 Occupational Therapist of Paraplegic Center Ostia, Ostia, Italy

3 Department of Anatomical, Histological, Forensic and Orthopaedic Sciences, "Sapienza" University of Rome, Rome, Italy

4 Department Human Neurosciences, Sapienza-University of Rome, IRCSS Neuromed Institute Pozzilli (IS), Pozzilli, Italy

5 Department Human Neurosciences, Sapienza-University of Rome, Rome, Italy

6 Department of Public Health and Infection Disease, Sapienza University of Rome, Rome, Italy

\section{Introduction}

The impairment of arm and hand function is one of the most disabling consequences of a spinal cord injury at the cervical level (C-SCI) because it greatly impacts a person's level of independence and quality of life [1,2]. Restoring arm and hand function is an important goal in rehabilitation and a specific priority for individuals with tetraplegia [3-5]. In rehabilitation, there are many evaluation scales for activities, from basic functions such as grasping and moving objects [6-11] to those that are more complex, such as getting dressed and using the toilet [12-15]. Compared with other tests, the advantage of the Van Lieshout Test (VLT) [15] is that it provides detailed information about how the tasks of the test are to be carried out, which makes it more useful in establishing treatment goals.

In 1994, the Hoensbroek Rehabilitation Center in the Netherlands began to write protocols to examine function of 
the hand in people with tetraplegia qualitatively. The protocol has undergone many changes and led to the VLT: the Van Lieshout hand function test for tetraplegia. The measurement concept of the VLT [15] takes into consideration and refers to the International Classification of Functioning, Disability, and Health (ICF) [16] and Nagi's Disability Model [17]. The goal of the Dutch group was to create an objective evaluation to measure the quality of movement of the upper limb in those with tetraplegia. The VLT consists of 19 items to assess the function of the arm and hand associated with activities of daily living, which best represents the level of activity in accordance with the ICF classification [16]. Performance levels were developed for each of the 19 tasks and a score of five corresponds to the best possible performance of an activity. The VLT Short Version (VLT-SV) [18] was developed in 2006 by Post et al. [18] to reduce the time of administration for clinical use. Before this work, the psychometric properties from only the long form were analyzed by researchers of the institute where the test was developed [19-21].

Since 2014, the Italian National Coordination of Professional Operators in Spinal Units has listed the VLT and the VLT-SV as important reference tools in their protocol for functional hand rehabilitation (http://www.cnopus.it/ilprotocollo-della-mano-funzionale/) and in the Italian medicine, physics, and rehabilitation guidelines (http://www. formazionesostenibile.it/2017/RomeRehabilitation/ libro_per_sito.pdf).

The cross-cultural adaptation of a measurement tool for use in multiple countries is essential. It is now recognized that if measurements are to be used across cultures, the items must be not only well-translated, but also culturally adapted so that the content validity of the instrument is maintained at a conceptual level from culture to culture. At the same time, a psychometric study is required for an outcome measure that contains subjective questions, as well as for a test that is conducted through observation. Language can affect the rater's interpretation of the information about how the tasks are to be carried out, which would be important to help standardize the results and make them reliable.

Recently, the long version of the VLT was validated in the Italian C-SCI population [22] to be used as a performance measure mainly for research purposes, while the short version, analyzed in the current study, could be more useful in clinical practice. To date the VLT-SV was used in many rehabilitation programs for individuals with C-SCI and we found many advantages with this test due to the detailed information it provides about how the tasks of the test are to be carried out. This makes it useful in creating a treatment regimen and in establishing treatment goals; moreover, the results are more standardized. However, the VLT-SV has never been evaluated in the Italian context.
Consequently, we decided to conduct this study to evaluate the psychometric properties of the VLT-SV in an Italian sample of people with C-SCI. This is also the first independent validation of the VLT-SV since the previous articles were written by researchers of the institute where the test was developed [18-21].

\section{Methods}

This study was conducted by a research group composed of medical doctors and rehabilitation professionals from the "Sapienza" University of Rome and from the "Rehabilitation \& Outcome Measure Assessment" (R.O.M.A.) association. In the last few years, the R.O.M.A. association has addressed validation of many outcome measures in Italy [22-32].

\section{Cultural adaptation procedures}

The version of the VLT adapted to Italian culture (IT-VLT) [22], developed in 2016, was used to create the Italian version of the short VLT (VLT-SV-IT) by removing the same items that were removed from the original VLT to create the VLT-SV [18, 22].

\section{Participants}

Consistent with the IT-VLT validation study [22], a different sample with the same characteristics was recruited for this study from three Italian spinal units. The sample inclusion criteria were:

- A-D level on the American Spinal Cord Injury Association Impairment Scale (AIS) [33].

- C-SCI (C3-T1 level).

- 18-65 years of age.

- The ability to sit in a wheelchair for at least three consecutive hours.

Exclusion criteria were C-SCI with severe additional diseases that interfered with the activities of daily living.

Consistent with the validation study of the long form [22], the sample needed to have at least 55 participants. Eligible participants were informed about the purpose, duration, and procedures of the study and those interested in taking part in the study gave their consent before inclusion in the scheduled testing session [34, 35]. In each participating rehabilitation center, according to a standardized protocol, one trained occupational therapist conducted the measurements. To measure the stability of the individual measurements carried out at different times (test-retest), the VLT-SV-IT was administered to the same sample after 
7 days. The Italian version of the Jebsen-Taylor Hand Function Test (JTHFT) [32] and the Italian version of the Spinal Cord Independence Measures (SCIM III) [36] were administered to a randomized subgroup of the sample.

\section{Instruments}

The VLT-SV [18] consists of 10 of the 19 items of the complete version and specifically evaluates the recovery of function and the functional state at the time of evaluation, with a total score ranging from 0 to 50 . The administration time of this tool is approximately $25 \mathrm{~min}$. The inability to perform a task may be due to the presence of limiting factors, either or both pathological (spasticity and joint limitations) or physiological (hand shape and arm length). The items of the VLT that are not present in the reduced version are the ability to propel a manual wheelchair; the ability to perform transfers; the ability to lift (e.g., to reduce the weight on the buttocks while seated); the ability to stabilize both arms against gravity; the ability to pick up an object from the ground; the ability to lift an object over the shoulders; hand sensitivity; thumb closure ability with wrist in extension; and the ability to close fingers with wrists in extension.

The JTHFT [5] is a seven-part, timed diagnostic test that assesses a person's level of hand function. Each subtest is designed to test each subject in precisely the same manner. The seven subtests include writing, simulated page turning, lifting small objects, simulated feeding, stacking, and lifting large, lightweight, and heavy objects. The JTHFT is administrated with a dynamometer to statistically record the force. The instrument used in the study was the Jamar J00105 [37], a dynamometer model that calculates the strength of the flexor muscles of the hand. The Italian version of the JTHFT [32] showed an intraclass correlation coefficient (ICC) for intra-rater and inter-rater reliability ranging from 0.282 to 0.695 for the dominant hand and 0.516-0.814 for the nondominant hand. Pearson's test revealed a strong correlation between all items and between the items and the gold standard (Jamar dynamometer), represented by the gripping force $(p<0.01$ and $p<0.05)$.

The SCIM was developed to address three specific areas of function in patients with SCI: self-care (feeding, grooming, bathing, and dressing); respiration and sphincter management; and an individual's mobility abilities (bed, transfers, and indoors/outdoors). The SCIM III was developed in 2002 as an international version of the prior version [9]. Catz et al. [10] designed this version to encompass individuals with SCI in every walk of life, regardless of their culture. The validity of the Italian version of the SCIM III [36] was confirmed by the close correlation with the functional independence measure results, both at admission and discharge $(r=0.91, p<0.01)$. Internal consistency, inter-rater reliability, and test-retest reliability were satisfactory overall, showing values higher than 0.90 .

\section{Statistical analyses/data analyses}

Consistent with the "COnsensus-based Standards for the selection of health Measurement INstruments" checklist recommendations (COSMIN) [38], we assessed the reliability and validity of the VLT-SV-IT as follows: Cronbach's alpha for internal consistency needed to be $>0.7$ to establish the degree of agreement between the various items; the ICC test-retest reliability needed to be $>0.7$ to establish the stability of the individual measurements carried out at different times; and Pearson's correlation coefficient between VLT-SV-IT and the Italian versions of the SCIM III and the JTHFT needed to be $>0.5$ or $<-0.5$, where positive values indicate positive linear correlation and negative values indicate negative linear correlation. The Pearson coefficient also has been analyzed to investigate the correlation between the VLT-SV-IT, the level of injury and the AIS [33]. The significance level was set for $\mathrm{p}$-value less than or equal to 0.05 . All statistical analyses were performed using IBM-SPSS version 23.00.

We certify that all applicable institutional and governmental regulations concerning the ethical use of human volunteers/animals were followed during the course of this research.

\section{Results}

\section{Participants}

From January to March 2018, 61 eligible people with acute and chronic SCI were recruited for this study. All of them agreed to participate and completed the study. Data on age, gender, years of injury, neurological classification of AIS [33], and dominant hand were collected from each participant. As reported in Table 1, 80.3\% of the sample was male and the average age was 47.23 years.

\section{Reliability and validity}

The total Cronbach's alpha was 0.95 for both the right and the left hand. Correlation of items showed statistically significant results with $p<0.01$ with a correlation from 0.94 to 0.95 for the right hand and 0.93 to 0.94 for the left hand; Cronbach's alpha values for each item are reported in Table 2. Seven days after the first administration, the VLTSV-IT was administered again to all participants and the ICC data showed statistically significant results with 0.90 for both the total VLT-SV-IT and the right and the left hand. The VLT-SV-IT demonstrated a good degree of concurrent 
Table 1 Demographic characteristics for the 61 participants in the reliability study of the VLT-IT-SV

\begin{tabular}{ll}
\hline & Sample $=61$ \\
\hline Age (mean \pm SD) & $47 \pm 14.76$ \\
Gender male number (\%) & $61(80)$ \\
AIS scale ${ }^{a}$ number (\%) & \\
A & $25(41)$ \\
B & $15(24)$ \\
C & $15(25)$ \\
D & $6(10)$ \\
Level of injury number (\%) & \\
C3 & $3(5)$ \\
C4 & $13(21)$ \\
C5 & $20(33)$ \\
C6 & $19(31)$ \\
C7 & $6(10)$
\end{tabular}

${ }^{\mathrm{a} A m e r i c a n}$ Spinal Injury Association Impairment Scale (AIS)

validity, showing several positive correlations with the Italian version of the JTHFT [10] and the dynamometer, as reported in Table 3. At the same time, correlation with the Italian version of the SCIM III [14] was poor, as reported in Table 4. Pearson's correlation coefficient with the level of injury and the AIS scale is also described in Table 4 [33].

\section{Discussion}

The functionality and treatment of hands in individuals with C-SCI have been the focus of much interest in the recent scientific literature [39-44]. This study analyzed the psychometric properties of VLT-SV and found it to be an optimal assessment tool for use in clinical practice. Based on the results, the VLT-SV-IT is a reliable, valid, and rapidly administrable outcome measure for assessing hand function in individuals with C-SCI. The Italian version of the manual will be available in accordance with the copyright owner's royalties (www.adelante-zorggroep.nl). Participants in the present study showed homogeneous characteristics and findings demonstrated that the assessment tool has good psychometric properties. Consistent with previous studies, the VLT-SV-IT has a high degree of internal consistency (Cronbach's alpha $=0.95$ ) and testretest reliability $(\mathrm{ICC}=0.90)$. The original VLT-SV has excellent internal consistency $($ Cronbach's alpha $=0.91$ ) and inter-rater reliability $(\mathrm{ICC}=0.98)$ as well [18].

The Pearson coefficient showed good results with the level of injury with a value of 0.51 . More precisely, it is an inversely proportional correlation: the higher the level of cervical lesion, the lower the VLT-SV-IT score. In contrast, the Pearson coefficient was poorly correlated with the AIS

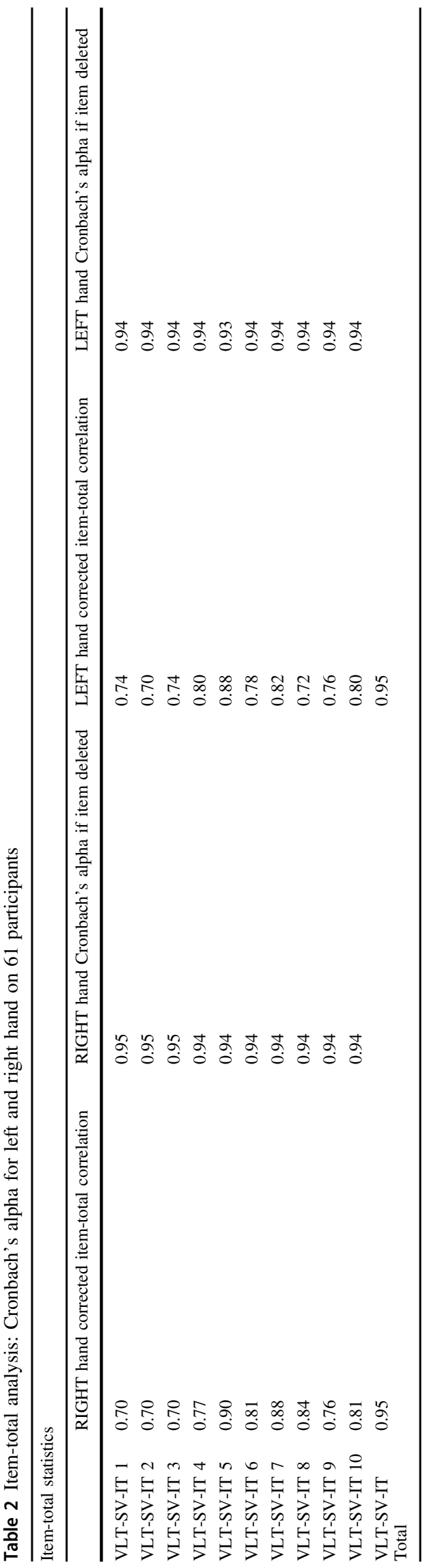




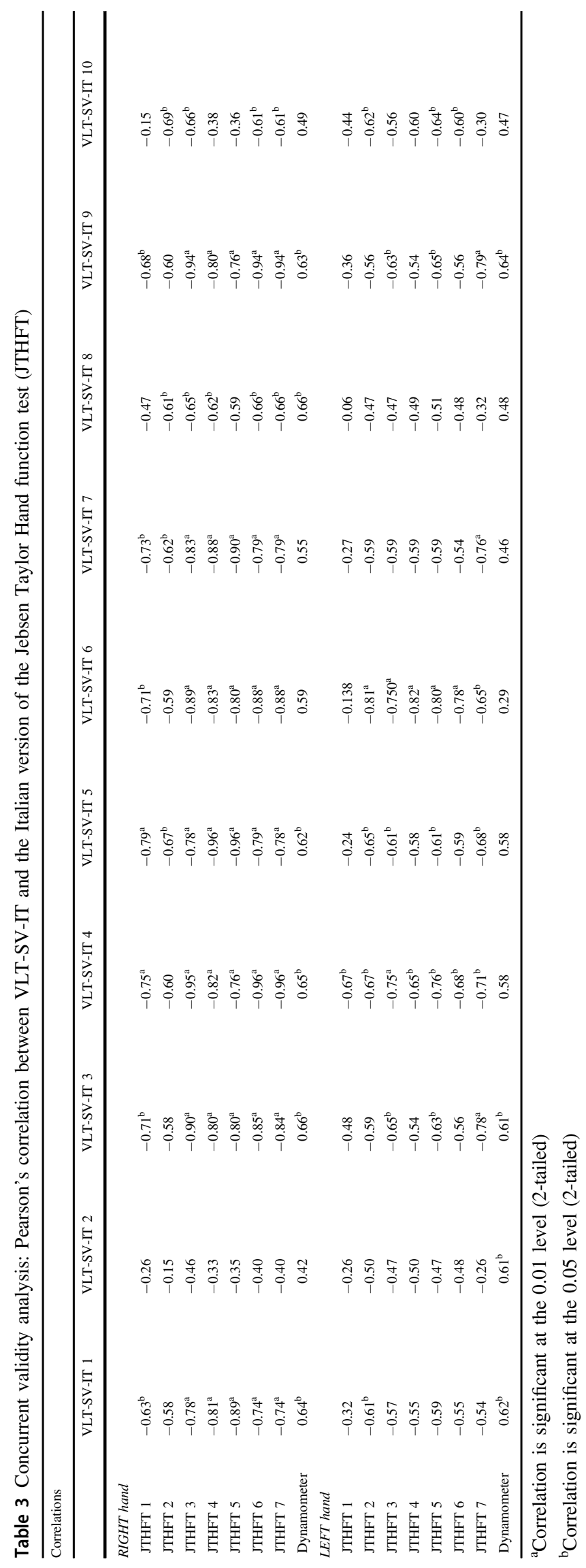

Table 4 Construct validity analysis: Pearson's correlation between VLT-SV-IT and level of injury, American Spinal Injury Association Impairment Scale (AIS) scale, and the Italian version of the Spinal Cord Independence Measure (SCIM III)

\begin{tabular}{llll}
\hline Correlations & & & \\
\hline & VLT-SV-IT & VLT-SV-IT & VLT-SV-IT \\
& right & left & total \\
Level of injury & $0.51^{\mathrm{a}}$ & $0.46^{\mathrm{a}}$ & $0.51^{\mathrm{a}}$ \\
AIS & 0.18 & 0.18 & 0.18 \\
SCIM III & 0.09 & 0.04 & 0.07 \\
\hline
\end{tabular}

${ }^{\mathrm{a}}$ Correlation is significant at the 0.01 level (2-tailed)

[33], showing that the completeness (sensory and motor level) of injury is not necessarily correlated with hand functionality. Regarding concurrent validity, in comparing the VLT with the JTHFT, it is possible to see that the correlations between the items of the right hand are stronger than those of the left hand. It is interesting to note how, for example, the VLT-SV item "Thumb socket," which evaluates fine motor skills, is strongly correlated with the JTHFT items "Writing," "Simulating turning a page," "Lifting small and light objects," "Simulating feeding," and "Stacking," which are all tasks that need suitable fine motor movements to be carried out. In the same way, the VLT-SV item "Strength of the thumb" is correlated with the JTHFT items "Writing," "Simulating feeding," "Stacking," and "Lifting large and light objects and large and heavy objects". The data also show how the VLT-SV item "Thumb opening" is strongly correlated with the JTHFT items "Lifting small and light objects," "Simulating feeding," "Lifting large objects and light objects," and "Lifting large and heavy objects" with a value of $-0.9,0.8,-0.8$, and -0.8 , respectively. The ability to open the fingers, as evaluated in the VLT-SV, is correlated with the ability to lift large, light, and heavy objects, as evaluated in the JTHFT. Finally, the strength of the fingers, as evaluated in the VLT-SV, correlates with the ability to simulate feeding and to lift large, light, and heavy objects evaluated in the JTHFT.

Consistent with the Italian validation study of the VLT long version [22], where there was excellent correlation with the SCIM III [36], we administered the VLT-SV-IT and the Italian version of the SCIM III [36] at the same time. However, in this study, the Pearson coefficient showed poor correlations with a score of 0.07 . By analyzing the data, we saw that among the nine tasks that were removed from the VLT to create the reduced version were the items "ability to propel a manual wheelchair"; "ability to perform transfers"; and "ability to lift (e.g., to reduce the weight on the buttocks while seated)." For the SCIM III-Italian version, nine items out of a total of 17 concern "Mobility and transfers." It is therefore clear why the correlation between SCIM III [36] and VLT-SV is not significant and why, in contrast, the correlation between VLT and SCIM III is very strong. 


\section{Limitations}

Consistent with the Italian validation study of the long version, the small sample size did not allow for an understanding of differences between participants with different levels of C-SCI. Furthermore, a larger group of participants probably would have given a better impression of the relationship between arm/hand impairment and the VLTSV-IT score. In future studies of the VLT-SV-IT, we recommend providing data about inter-rater reliability to be consistent with the validation study of the long version. Finally, the VLT is not one of the most widely used instruments to assess hand function, but we highly recommend it because of the detailed information it provides about how the tasks of the test are to be carried out, which might make it more useful in establishing treatment goals.

\section{Conclusions}

The present study gives evidence about the reliability, validity, rapidity of administration, and ease of scoring of the VLTSV for health professionals and researchers to investigate and explicate hand function in the person with tetraplegia in a reproducible way. The results confirm that it could be used as a starting point for hand function therapy, as a predictor of future independence in the activities of daily living, and to assist in clinical decision-making regarding treatment policy. Given the great usefulness and practicality of this short version in evaluating hand function in individuals with C-SCI, we hope that our study is a starting point and that it encourages rehabilitators worldwide to validate the test in their own cultural context so that it can become a reference measure in assessing hand function in individuals with C-SCI.

\section{Data availability}

The datasets generated and/or analysed during the current study are available from the corresponding author on reasonable request.

Acknowledgements We acknowledge those who participated in the surveys; their collective expertise greatly informed the development of the Italian version of the VLT.

Author contributions BA, GG, and MMA: Conceived and/or designed the work that led to the submission, acquired data, and/or played an important role in interpreting the results. BA and TM: Drafted or revised the manuscript. DSR, FG, and VD: Approved the final version.

\section{Compliance with ethical standards}

Conflict of interest The authors declare that they have no conflict of interest.
Ethical approval We certify that all applicable institutional and governmental regulations concerning the ethical use of human volunteers were followed during the course of this research. All procedures followed were in accordance with the ethical standards of the responsible committee on human experimentation (institutional and national) and with the Helsinki Declaration of 1975, as revised in 2008

Informed consent Informed consent was obtained from all participants for being included in the study.

\section{References}

1. Snoek GJ, IJzerman MJ, Hermens HJ, Maxwell D, BieringSorensen F. Survey of the needs of patients with spinal cord injury: impact and priority for improvement in hand function in tetraplegics. Spinal Cord. 2004;42:526-32.

2. Simpson LA, Eng JJ, Hsieh JT, Wolfe DL. The health and life priorities of individuals with spinal cord injury: a systematic review. J Neurotrauma. 2012;29:1548-55.

3. Franke AC, Snoek GJ, de Groot S, Nene AV, Spooren AI, Post MW. Arm hand skilled performance in persons with a cervical spinal cord injury-long-term follow-up. Spinal Cord. 2013;51:161-4.

4. Biering-Sørensen F, Bickenbach JE, El Masry WS, Officer A, von Groote PM. ISCoS-WHO collaboration. International Perspectives of Spinal Cord Injury (IPSCI) report. Spinal Cord. 2011;49:679-83.

5. Lu X, Battistuzzo CR, Zoghi M, Galea MP. Effects of training on upper limb function after cervical spinal cord injury: a systematic review. Clin Rehabil. 2015;29:3-13.

6. Hsieh CL, Hsueh IP, Chiang FM, Lin PH. Inter-rater reliability and validity of the action research arm test in stroke patients. Age Ageing. 1998;27:107-13.

7. Jebsen RH, Taylor N, Trieschmann RB, Trotter MJ, Howard LA. An objective and standardized test of hand function. Arch Phys Med Rehabil. 1969;50:311-9.

8. Hudak PL, Amadio PC, Bombardier C. Development of an upper extremity outcome measure: the DASH. Am J Ind Med. 1996;29:602-8.

9. Land NE, Odding E, Duivenvoorden HJ, Bergen MP, Stam HJ. Tetraplegia Hand Activity Questionnaire (THAQ): the development, assessment of arm-hand function-related activities in tetraplegic patients with a spinal cord injury. Spinal Cord. 2004;42:294-301.

10. Sollerman C, Ejeskär A. Sollerman hand function test. A standardized method and its use in tetraplegic patients. Scand J Plast Reconstr Surg Hand Surg. 1995;29:167-76.

11. Kalsi-Ryan S, Curt A, Verrier MC, Fehlings MG. Development of the Graded Redefined Assessment of Strength, Sensibility and Prehension (GRASSP): reviewing measurement specific to the upper limb in tetraplegia. J Neurosurg Spine. 2012;17:65-76.

12. Catz A, Itzkovich M, Agranov E, Ring H, Tamir A. SCIMspinal cord independence measure: a new disability scale for patients with spinal cord lesions. Spinal Cord. 1997;35:850-6.

13. Keith RA, Granger CV, Hamilton BB, Sherwin FS. The functional independence measure: a new tool for rehabilitation. Adv Clin Rehabil. 1987;1:6-18.

14. Gresham GE, Labi ML, Dittmar SS, Hicks JT, Joyce SZ, Stehlik MA. The Quadriplegia Index of Function (QIF): sensitivity and reliability demonstrated in a study of thirty quadriplegic patients. Paraplegia. 1986;24:38-44.

15. Van Lieshout, G User manual Van Lieshout Test. (2003).

16. Kostanjsek N. Use of The International Classification of Functioning, Disability and Health (ICF) as a conceptual framework 
and common language for disability statistics and health information systems. BMC Public Health. 2011;11(Suppl 4):S3 https:// doi.org/10.1186/1471-2458-11-S4-S3.

17. Nagi SZ. A study in the evaluation of disability and rehabilitation potential: concepts, methods, and procedures. Am J Public Health Nations Health. 1964;54:1568-79.

18. Post MW, Van Lieshout G, Seelen HA, Snoek GJ, Ijzerman MJ, Pons C. Measurement properties of the short version of the Van Lieshout test for arm/hand function of persons with tetraplegia after spinal cord injury. Spinal Cord. 2006;44:763-71.

19. Spooren AI, Arnould C, Smeets RJ, Bongers HM, Seelen HA. Improvement of the Van Lieshout hand function test for tetraplegia using a Rasch analysis. Spinal Cord. 2013;51:739-44. https://doi.org/10.1038/sc.2013.54

20. Spooren AI, Arnould C, Smeets RJ, Snoek G, Seelen HA. Reference values for the transformed Van Lieshout hand function test for tetraplegia. Spinal Cord. 2013;51:745-9. https://doi.org/ 10.1038/sc.2013.73.

21. Spooren AI, Janssen-Potten YJ, Post MW, Kerckhofs E, Nene A, Seelen HA. Measuring change in arm hand skilled performance in persons with a cervical spinal cord injury: responsiveness of the Van Lieshout Test. Spinal Cord. 2006;44:772-9.

22. Galeoto G, Berardi A, De Santis R, Di Valentini L, Beccasio R, Marquez MA, et al. Validation and cross-cultural adaptation of the Van Lieshout test in an Italian population with cervical spinal cord injury: a psychometric study. Spinal Cord Ser Cases. 2018;4:49.

23. Marquez MA, De Santis R, Ammendola V, Antonacci M, Santilli $\mathrm{V}$, Berardi A, et al. Cross-cultural adaptation and validation of the "Spinal Cord Injury-Falls Concern Scale" in the Italian population. Spinal Cord 2018;1. https://doi.org/10.1038/s41393-0180070-6.

24. Galeoto G, Colalelli F, Massai P, Berardi A, Tofani M, Pierantozzi M, et al. Quality of life in Parkinson's disease: Italian validation of the Parkinson's Disease Questionnaire (PDQ-39-IT). Neurol Sci 2018 https://doi.org/10.1007/s10072-018-3524-x.

25. Covotta A, Gagliardi M, Berardi A, Maggi G, Pierelli F, Mollica $\mathrm{R}$, et al. Physical activity scale for the elderly: translation, cultural adaptation, and validation of the Italian Version. Curr Gerontol Geriatr Res. 2018;2018:8294568 https://doi.org/10.1155/2018/ 8294568

26. Galeoto G, Sansoni J, Scuccimarri M, Bruni V, De Santis R, Colucci $\mathrm{M}$, et al. A psychometric properties evaluation of the italian version of the geriatric depression scale. Depress Res Treat. 2018;2018:1797536 https://doi.org/10.1155/2018/1797536

27. Murgia M, Bernetti A, Delicata M, Massetti C, Achilli EM, Mangone M, et al. Inter-and intra-interviewer reliability of Italian version of Pediatric Evaluation of Disability Inventory. Ann Ig. 2018;30:153-61. https://doi.org/10.7416/ai.2018.2206.

28. Berardi A, De Santis R, Tofani M, Marquez MA, Santilli V, Rushton PW, et al. The Wheelchair Use Confidence Scale: Italian translation, adaptation, and validation of the short form. Disabil Rehabil Assist Technol. 2018;13:575-80. https://doi.org/10.1080/ 17483107.2017.1357053

29. Tofani M, Candeloro C, Sabbadini M, Lucibello L, Figura M, Fabbrini G, et al. The psychosocial impact of assistive device scale: Italian validation in a cohort of nonambulant people with neuromotor disorders. Assist Technol 2018:1-6. https://doi.org/ 10.1080/10400435.2018.1469553.

30. Castiglia SF, Galeoto G, Lauta A, Palumbo A, Tirinelli F, Viselli $\mathrm{F}$, et al. The culturally adapted Italian version of the Barthel Index
(IcaBI): assessment of structural validity, inter-rater reliability and responsiveness to clinically relevant improvements in patients admitted to inpatient rehabilitation centers. Funct Neurol. 2017;22:221-8.

31. Galeoto G, Lauta A, Palumbo A, Castiglia SF, Mollica R, SantillI V. The Barthel Index: Italian translation, adaptation and validation. Int J Neurol Neurother. 2015;2:1-7. https://doi.org/10.23937/ $2378-3001 / 2 / 2 / 1028$

32. Culicchia G, Nobilia M, Asturi M, Santilli V, Paoloni M, De Santis R, et al. Cross-cultural adaptation and validation of the Jebsen-Taylor Hand Function Test in an Italian population. Rehabil Res Pract. 2016;2016:8970917 https://doi.org/10.1155/ 2016/8970917

33. Kirshblum SC, Burns SP, Biering-Sorensen F, Donovan W, Graves DE, Jha A, et al. International standards for neurological classification of spinal cord injury (Revised 2011). J Spinal Cord Med. 2011;34:535-46. https://doi.org/10.1179/ 204577211 X13207446293695

34. Galeoto G, De Santis R, Marcolini A, Cinelli A, Cecchi R. Il consenso informato in Terapia Occupazionale: proposta di una modulistica. G Ital Med Lav Ergon. 2016;38:107-15.

35. Galeoto G, Mollica R, Astorino O, Cecchi R. Informed consent in physiotherapy: proposal of a form. G Ital Med Lav Ergon. 2015;37:245-54.

36. Invernizzi M, Carda S, Milani P, Mattana F, Fletzer D, Iolascon $\mathrm{G}$, et al. Development and validation of the Italian version of the Spinal Cord Independence Measure III. Disabil Rehabil. 2010;32:1194-203.

37. Lafayette Instrument Company, Inc. (2004). Lafayette Instrument JAMAR Hydrolic Hand Dynamometer User Instructions Model J00105. Europe. https://www.chponline.com/store/pdfs/j-20.pdf

38. Mokkink LB, Terwee CB, Patrick DL, Alonso J, Stratford PW, Knol DL, et al. The COSMIN checklist for assessing the methodological quality of studies on measurement properties of health status measurement instruments: an international Delphi study. Qual Life Res. 2010;19:539-49. https://doi.org/10.1007/s11136010-9606-8

39. Thorsen R, Binda L, Chiaramonte S, Dalla Costa D, Redaelli T, Occhi E, et al. Correlation among lesion level, muscle strength and hand function in cervical spinal cord injury. Eur J Phys Rehabil Med. 2014;50:31-8.

40. Yozbatiran N, Keser Z, Hasan K, Stampas A, Korupolu R, Kim S, et al. White matter changes in corticospinal tract associated with improvement in arm and hand functions in incomplete cervical spinal cord injury: pilot case series. Spinal Cord Ser Cases. 2017;3:17028.

41. Lim M, Park J, Lee KJ, Kweon H, Kim B, Cho K, et al. Usability of a new writing assistive device for persons with cervical spinal cord injury. Stud Health Technol Inform. 2015;217:710-7.

42. Francisco GE, Yozbatiran N, Berliner J, O'Malley MK, Pehlivan AU, Kadivar Z, et al. Robot-assisted training of arm and hand movement shows functional improvements for incomplete cervical spinal cord injury. Am J Phys Med Rehabil. 2017;96(10 Suppl 1):S171-S177.

43. Lei Y, Perez MA. Phase-dependent deficits during reach-to-grasp after human spinal cord injury. J Neurophysiol. 2018;119:251-61.

44. Harvey LA, Glinsky JV, Bowden JL. The effectiveness of 22 commonly administered physiotherapy interventions for people with spinal cord injury: a systematic review. Spinal Cord. 2016;54:914-23. 